\title{
Anaplastic Medulloblastoma
}

National Cancer Institute

\section{Source}

National Cancer Institute. Anaplastic Medulloblastoma. NCI Thesaurus. Code $C 92625$.

A medulloblastoma characterized by marked nuclear pleomorphism, and high mitotic activity. 